OPEN ACCESS

Edited by:

Xiaoli Bing,

Nanjing Agricultural University, China

Reviewed by:

Mohammad Mehrabadi,

Tarbiat Modares University, Iran

Jia Wang,

Southwest University, China

${ }^{*}$ Correspondence:

Bao-Li Qiu

baileyqiu@scau.edu.cn

Specialty section:

This article was submitted to

Invertebrate Physiology,

a section of the journal

Frontiers in Physiology

Received: 16 September 2021 Accepted: 11 November 2021

Published: 10 December 2021

Citation:

Zhang L-H, Ren S-L, Su Z-Q, Xu P-P, Ou D, Wang L-J, Sang $W$ and Qiu B-L (2021) Impact

of Huanglongbing Pathogen Infection

on the Amino Acid Composition in Both Citrus Plants and the Asian Citrus Psyllid.

Front. Physiol. 12:777908. doi: 10.3389/fphys.2021.777908

\section{Impact of Huanglongbing Pathogen Infection on the Amino Acid Composition in Both Citrus Plants and the Asian Citrus Psyllid}

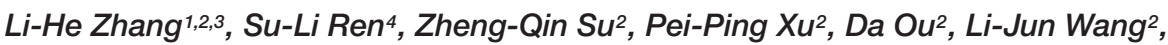 \\ Wen Sang ${ }^{2}$ and Bao-Li Qiu ${ }^{1,2 *}$ \\ ${ }^{1}$ Chongqing Key Laboratory of Vector Insects, College of Life Sciences, Chongqing Normal University, Chongqing, China, \\ 2 Guangdong Laboratory for Lingnan Modern Agriculture, Guangzhou, China, ${ }^{3}$ Guangdong Laboratory of Lingnan Modern \\ Agriculture, Genome Analysis Laboratory of the Ministry of Agriculture and Rural Affairs, Agricultural Genomics Institute \\ at Shenzhen, Chinese Academy of Agricultural Sciences, Shenzhen, China, ${ }^{4}$ Airport Management College, Guangzhou Civil \\ Aviation College, Guangzhou, China
}

The Asian citrus psyllid (ACP) Diaphorina citri is the main vector of the pathogen Candidatus Liberibacter asiaticus (CLas), which is the causal agent of citrus Huanglongbing disease. Feeding by both ACP nymphs and adults on host plants allows them to obtain nutrition. Therefore, the nutritional content within the plant phloem is of much importance for the development and reproduction of ACP. The infection by pathogenic microbiomes may affect the amino acid contents of their host plants and then indirectly affect the biology of sap-feeding insects. In this study, we investigated the amino acid contents and their proportions in both CLas-infected and CLas-free citrus plants, ACP adults, and also in honeydew produced by ACP nymphs. Results showed that infection by CLas had a large impact on the amino acid species and proportion in all the tested target plants, ACP adults, and in the honeydew of ACP nymphs. The content of total amino acids in CLas-infected citrus was much higher than that of CLasfree citrus. However, CLas infection significantly reduced the proportion of essential amino acids (EAAs) in these plants. When feeding on CLas-infected citrus plants, ACP adults absorbed less total amino acids than those adults feeding on healthy plants, but the proportion of EAAs was significantly higher when they fed on CLas-infected citrus plants. The proportion of EAAs also significantly increased in the honeydew secreted by ACP nymphs that fed on CLas-infected citrus plants. However, EAA detection in the honeydew of ACP nymphs indicated that the utilization rate of EAAs by CLas positive ACP nymphs was reduced. Our study has revealed that CLas infection significantly affects the contents, proportion, and utilization efficiency of different amino acids in citrus plants, ACP adults, and nymphs, leading to a developmental pattern of ACP that is more conducive to CLas transmission.

Keywords: Asian citrus psyllid, citrus huanglongbing, phloem sap, amino acids, honeydew 


\section{INTRODUCTION}

Huanglongbing (HLB), commonly known as citrus greening disease, is caused by three species of the fastidious, phloemresiding, gram-negative bacteria "Candidatus Liberibacter asiaticus” (CLas; Bové, 2006), “Ca. L. africanus” (CLaf; Garnier et al., 2000), and "Ca. L. americanus" (CLam; Texeira et al., 2005). It is one of the most economically destructive diseases of the citrus industry worldwide (Wang et al., 2016). At present, there is no established cure for this century-old disease. HLB, whose name in Chinese means "yellow dragon disease," was first reported from southern China in 1919 but is thought to most likely have originated in Taiwan Island in the 1870s. At present, it is now known to occur in approximately 40 different countries across Asia, Africa, Oceania, North America, and South America (Bové, 2006; Belasque et al., 2010).

The HLB pathogen resides in the phloem of host plants and causes a systemic disease (Jagoueix et al., 1994), for example, it has caused crippling diseases denoted "greening" in South Africa, "mottle-leaf" in the Philippines, "dieback" in India, and "vein phloem degeneration" in Indonesia (Bové, 2006). The HLBinfected trees display a blotchy mottle condition of the leaves that results in the development of yellow shoots, which is the early and typical characteristic symptom of the disease. The trees then gradually become stunted, bearing only a few small-sized and deformed (lop-sided) fruits, which are poorly colored (greening). The fruit coloration starts at the peduncular end (color inversion) and results in fruit loss and eventual tree death (Roistacher, 1996; Bové, 2006).

The amino acid content in the phloem sap of host plants is the important nutritional component for the successful development and reproduction of piercing-sucking insects such as whitefly, psyllids, aphids, white wax scale, plant hopper, and leafhoppers; these insects take amino acids directly from leaf liquid instead of proteins (Ding, 1990; Zhao et al., 2001). It is thought that pathogenic microbiomes may affect the amino acid content of host plants and, as a result, indirectly affect the behavior of insects feeding on the plants. For example, it has been found that cassava plants infected with East African cassava mosaic virus (EACMV) can significantly increase the population growth of the whitefly Bemisia tabaci. It was deduced that this virus perhaps improves the contents of Glutamine, Aspartic acid (Asp), Tryptophan (Trp), and Tyrosine (Tyr) in the phloem sap of cassava plants (Colvin et al., 2006). Jiu et al. (2007) found that in comparison with its performance on healthy tobacco, $B$. tabaci showed a significantly increased fecundity and longevity through feeding on tobacco plants infected with two begomoviruses. Other studies have also shown that the aphid Aphis gossypii feeding on Zucchini yellow mosaic virus-infected pumpkin can increase their fecundity and longevity. Thus, it is speculated that the increase of fecundity and longevity may be closely related to the higher amino acid concentration in the phloem sap of pathogen-infected host plants (Blua et al., 1994; Stout et al., 2006).

The Asian citrus psyllid (ACP), Diaphorina citri Kuwayama (Hemiptera, Liviidae), is the main vector of bacteria in the genus Liberibacter, which are the causal agents of HLB (Tsai et al., 1988; Onagbola et al., 2011). Amino acids required by most insects are obtained from food proteins. However, some piercing-sucking insects, such as white wax scale, plant hopper, and leafhopper, directly take amino acids from the host leaf liquid instead of those via proteins (Ding, 1990; Zhao et al., 2001). ACP immatures and adults need to assimilate nutrition from host plants for their successful growth and development. There are studies showing that aphids increase their own fecundity after feeding on susceptible host plants. It could be speculated that the increase in fecundity may be closely related to an increase of some amino acids in the phloem sap of the susceptible host plant (Holopainen et al., 1997; Sandström, 2000; Walter and Difonzo, 2014). Ren et al. (2016) revealed that the infection of CLas both in citrus shoots and ACP produced a population increase of ACP. However, the mechanisms underlying the nutrition-related interactions among CLas pathogen, host plants (especially with respect to amino acid nutrition), and ACP are not completely understood.

Therefore, it is necessary to explore the link between the free amino acids (FAAs), essential amino acids (EAAs) required by ACP, and the phloem amino acid composition of host plants after infection with CLas pathogen. In this study, we compared the changes of total FAA content and their proportions in different treatments (CLas-infected and CLas-free citrus plants, CLaspositive and -negative ACP, and CLas-positive and -negative honeydew). The purpose of the study was to clarify the nutritional relationship between the HLB pathogen, citrus host plants, and the vector insect ACP. From the perspective of amino acid utilization, the effect of the HLB pathogen on the absorption and utilization of amino acids by the ACP was specifically considered.

\section{MATERIALS AND METHODS}

\section{Host Plants}

The HLB-free and HLB-infected citrus trees "Shatangju" (Citrus flamea Hort. ex Tseng shiyueju) were first selected from two different citrus groves in Zhaoqing, Guangdong, China in November 2017. These trees were completely removed, reinstated, watered, and fertilized as required in the experimental field site of the campus of South China Agricultural University (SCAU), Guangzhou, China. Nested quantitative PCR (qPCR) detection was performed periodically to detect the CLas infection in these citrus plants using the method described by Coy et al. (2014). In brief, the nested PCR increases the specificity and sensitivity of detection. Nested PCR uses two pairs of PCR primers to amplify the complete fragment. In the first step, the target DNA is combined with the first pair of common primers. In the next step, the second set of nested primers (inside the first PCR amplification fragment) is used to perform the second PCR on the products amplified by the first round of PCR. The first pair of primers used for CLas detection was 5'-AAGGAGGTGATCCAGCCGC-3', 27F: 5'-AGAGTTTGATCATGGCTCAG-3' in this study. The PCR procedure entailed pre-denaturation at $94^{\circ} \mathrm{C}$ for $5 \mathrm{~min}$ then followed by 20 cycles of $94^{\circ} \mathrm{C}$ for $30 \mathrm{~s}, 50^{\circ} \mathrm{C}$ for $30 \mathrm{~s}$, and $72^{\circ} \mathrm{C}$ for $1 \mathrm{~min}$, and finally a 4 -min extension period at $72^{\circ} \mathrm{C}$. The total system for the first round of 
PCR amplification is $25 \mu \mathrm{l}$, including $2.5 \mu \mathrm{l}$ PCR buffer, $2.5 \mu \mathrm{l}$ dNTPs, $0.5 \mu \mathrm{l}$ upstream and downstream primers, respectively, $0.4 \mu \mathrm{l}$ Taq enzyme, $1 \mu \mathrm{l}$ DNA template, and $17.6 \mu \mathrm{lddH} 2 \mathrm{O}$. The second pair of primers used for CLas detection was OI1: 5'-GCGCGTATGCAATACGAGCGGCA$3^{\prime}$ and OI2c: 5'-GCCTCGCGACTTCGCAACCCAT- $3^{\prime}$. The PCR procedure entailed pre-denaturation at $96^{\circ} \mathrm{C}$ for $1 \mathrm{~min}$ then followed by 35 cycles of $94^{\circ} \mathrm{C}$ for $30 \mathrm{~s}, 55^{\circ} \mathrm{C}$ for $30 \mathrm{~s}$, and $72^{\circ} \mathrm{C}$ for $1 \mathrm{~min}$, and finally a 4 -min extension period at $72^{\circ} \mathrm{C}$. The total system for the second round of PCR amplification is $25 \mu \mathrm{l}$, including $2.5 \mu \mathrm{l}$ PCR buffer, $2.5 \mu \mathrm{l}$ dNTPs, $0.5 \mu \mathrm{l}$ upstream and downstream primers, respectively, $0.4 \mu \mathrm{l}$ Taq enzyme, $2 \mu \mathrm{l}$ DNA template, and $16.6 \mu 1 \mathrm{ddH} 2 \mathrm{O}$.

\section{Insects}

The CLas-negative colony of the ACP was originally collected from HLB-free plants of Murraya paniculata (L.) in field sites belonging to SCAU in 2017. Following collection, the ACP was tested for CLas to ensure that it was a CLasnegative population. The ACP population was continuously reared on the young shoots of healthy citrus plants. The CLaspositive colony of ACP was set up by allowing ACP adults to reproduce a new generation on CLas-infected citrus shoots. Similarly, the infection status of ACP adults was monitored periodically by nested-qPCR according to the description of Coy et al. (2014). Both the CLas-positive and CLas-negative ACP populations were reared for 10 generations on these HLB-free and HLB-infected citrus trees, respectively, before being used in experimental trials.

\section{Phloem Sap Collection}

To collect the phloem sap exudates of a citrus plant, CLas-infected and CLas-free citrus plants were watered and fertilized and then pruned to encourage new shoot growth. Three shoots with 2-3 pieces of unfolded leaves of each kind of plant were quickly cut with an operating knife blade and inserted into a centrifuge tube with $2 \mathrm{ml}$ of ethylenediaminetetraacetic acid (EDTA) solution $(0.02 \mathrm{~mol} / \mathrm{L}$, $\mathrm{pH}$ 7.0). The samples were then placed in the dark at $26^{\circ} \mathrm{C}, \mathrm{RH}>90 \%$ for $12 \mathrm{~h}$. Following this, the citrus shoots were removed, and the solution was diluted to $4 \mathrm{ml}$ with EDTA and subsequently filtered with a syringe filter and conserved at $-20^{\circ} \mathrm{C}$ for experimental use (Su et al., 2015; Guo et al., 2019).

\section{Asian Citrus Psyllid Honeydew Collection}

To collect the honeydew, a dustpan-shaped box made of tinfoil was fixed under the tender branches where ACP nymphs were abundantly fed. As a result, the honeydew secreted by the ACP nymphs fell onto the tinfoil container. The whole branch and tinfoil box were covered with nylon bags to avoid the interference of other insects, such as ants. Following 1 week, the honeydew on the dustpan-shaped tinfoil box was gently collected into 1.5$\mathrm{ml}$ centrifugal tubes and carefully weighed before being stored at $-20^{\circ} \mathrm{C}$ for experimental trials.

\section{Amino Acid Analyses}

Free Amino Acids From Phloem Sap and Asian Citrus Psyllid Nymph Honeydew

The collected phloem sap exudates of CLas-infected and CLasfree citrus trees, and the collected ACP nymph honeydew was first diluted with $1 \mathrm{ml} 8 \%$ sulfosalicylic acid. Then, $100 \mu \mathrm{l}$ of diluent and FAA standard solutions were put into $1.5-\mathrm{ml}$ centrifugal tubes, respectively. Then, the same volume of ninhydrin and its buffer were added into each centrifugal tube and boiled for $5 \mathrm{~min}$. The color of phloem sap exudate and honeydew solutions was observed and diluted with $6 \%$ sulfosalicylic acid as required until their color was close to the FAA standard solution. Both solutions were then filtered through a $0.45-\mu \mathrm{m}$ filter. The FAA contents were analyzed using an amino acid analyzer S433 (Sykam, Munich, Germany), and all the analyses were performed with three biological replicates.

\section{Free Amino Acids From Asian Citrus Psyllid Adults}

The examination of FAAs was performed as described by Pan et al. (2013). In brief, approximately 80 ACP adults (7 days old) were collected from HLB-free and HLB-infected citrus trees, respectively. They were first ground in liquid nitrogen, dissolved

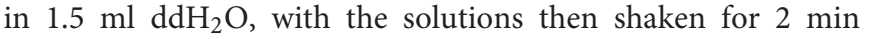
on a vortex shaker, and centrifuged at $14,000 \mathrm{rpm}$ for $10 \mathrm{~min}$. Following this, the supernatant was successively mixed with n-hexane, sulfosalicylic acid, and sodium citrate buffer solution, centrifuged at 10,000 rpm for $10 \mathrm{~min}$ between each two mixture treatments, before finally being passed through a $0.45-\mu \mathrm{m}$ filter. As with the FAA analysis in the phloem sap exudate, the FAA contents in ACP adults were analyzed using an amino acid analyzer S433 (Sykam, Munich, Germany), and all the analyses were performed with three biological replicates.

\section{Statistical Analyses}

The FAAs were determined by the automatic amino acid analyzer. The percentage of the various amino acids was calculated by the following formula: the respective amino acid content/the total amino acid content $\times 100 \%$. The contents of total amino acids and the percentage of EAAs and non-essential amino acids (NEAAs) were analyzed using SPSS 19.0 (for Windows; SPSS, Chicago, IL, United States), and the differences were compared using the $t$-test at a significant level of $P<0.05$.

\section{RESULTS}

\section{The Contents of Free Amino Acids in the Phloem Sap of Candidatus Liberibacter Asiaticus-Infected and Candidatus Liberibacter Asiaticus-Free Citrus}

According to the results of the determination of FAAs in the phloem sap, the contents of total amino acids in the phloem of CLas-infected citrus were significantly higher than that of CLas-free citrus ( $t=42.401, \mathrm{df}=2, P<0.001)$ (Figure 1A). In this study, we selected the same species of amino acids detected in the phloem sap of CLas-infected and CLas-free citrus plants 


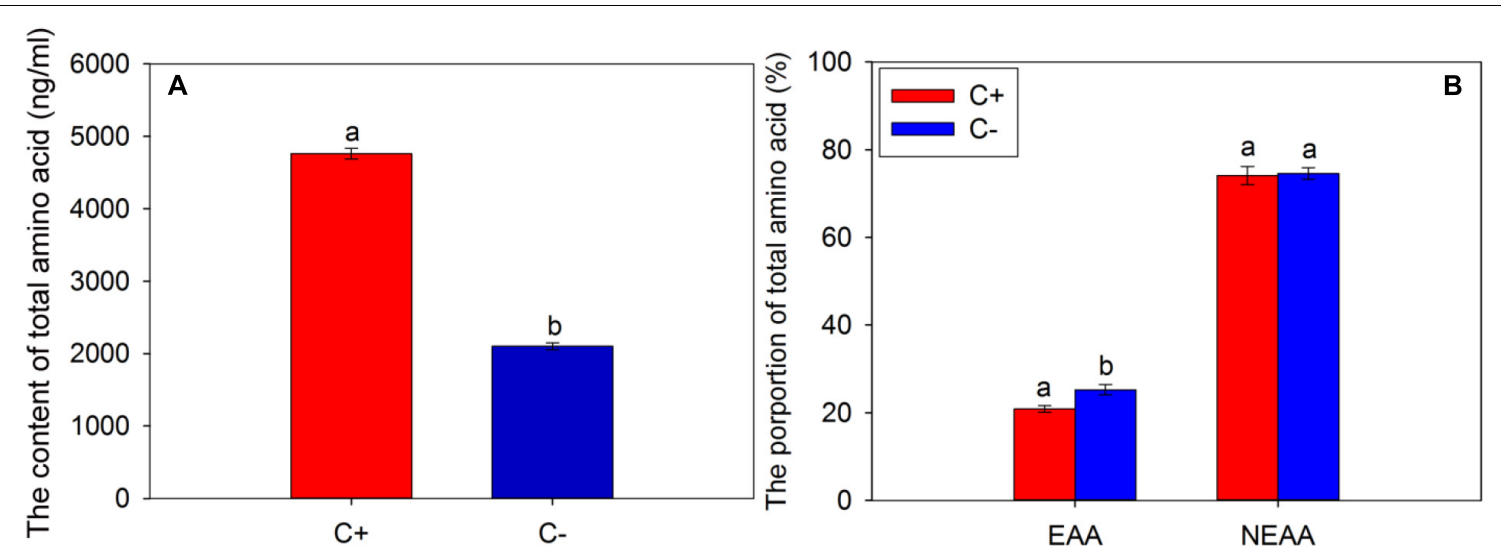

FIGURE 1 | The contents of a total amino acid (A) and the proportions of essential amino acids (EAAs) and non-essential amino acids (NEAAs) (B) in the phloem sap of citrus plants. "C+" and "C-" represent Candidatus Liberibacter asiaticus (CLas)-infected and CLas-free citrus plants, respectively; EAA, essential amino acid; NEAA, non-essential amino acid. Values are represented as mean $\pm \mathrm{SE}$, and different letters over the bars mean significant differences between groups at $P<0.05$

for analysis and found that the proportion of EAAs in the CLas-infected citrus plants was significantly lower than that of the CLas-free citrus plants $(t=-18.395$, df $=2, P=0.003)$ (Figure 1B), which means the infection of CLas significantly reduced the proportion of EAAs in the CLas-infected citrus plants $(t=-18.395$, $\mathrm{df}=2, P=0.003)$, but no significant difference was found between the NEAA proportions of CLas infection and CLas-free citrus plants $(t=-2.014$, df $=2$, $P=0.182)$.

\section{Free Amino Acid Composition in the Phloem Sap of Citrus Plants}

A total of 24 FAAs were detected in both CLas-infected and CLas-free citrus plants, with NEAAs being the main amino acid components in the phloem sap of citrus plants. However, CLas infection significantly affected the contents of 21 amino acids except Leucine (Leu), Glycine (Gly), and Tyr. For example, CLas infection greatly increased the mass percentage of Asparagine $\left(\mathrm{AspNH} \mathrm{N}_{2}\right)$ and Proline (Pro) but decreased the mass percentage of Arginine (Arg), Alanine (Ala), Asp, $\gamma$-aminobutyric acid (g$\mathrm{ABA})$, Ammonia $\left(\mathrm{NH}_{3}\right)$, Phosphatidylserine (P-ser), and Serine (Ser) in the phloem sap of CLas-infected citrus plants (Figure 2). The HLB pathogen, therefore, has a significant influence on the proportion of most components of EAAs and NEAAs in the host plant $(P<0.05)$.

\section{The Contents of Free Amino Acids in Asian Citrus Psyllid Adults}

The CLas infection greatly impacted the contents of total FAAs of ACP adults. Compared with the ACP adults that fed on CLas-free citrus plants, the contents of total amino acids of ACP adults that fed on CLas-infected citrus decreased significantly (Figure 3A) $(t=-18.395, \mathrm{df}=2, P=0.003)$. In this study, we selected the same species of amino acids detected in the CLas-positive and CLasnegative ACP populations for analysis and found that the EAA proportion of the ACP adults feeding on CLas-infected citrus plants was significantly higher than that of the ACP adults feeding on CLas-free citrus plants ( $t=24.861, \mathrm{df}=2, P=0.002)$, while the proportion of NEAAs showed the opposite trend $(t=-94.353$, $\mathrm{df}=2, P<0.001$ ) (Figure 3B). These results may reflect that the ACP adults that fed on CLas-infected citrus plants absorbed more EAAs than those ACP adults feeding on CLas-free citrus plants.

\section{Free Amino Acid Composition in the Asian Citrus Psyllid Adults}

The same total of 26 FAAs was identified in the ACP adults that fed on both CLas-free and CLas-infected citrus plants. CLas infection significantly influenced the proportion of the EAAs especially Arg, Histidine (His), and Threonine (Thr), and the NEAAs, in particular, Ala, L-isoglutamine $\left(\mathrm{GluNH}_{2}\right)$, Pro, and Tyr $(P<0.05)$ (Figure 4).

\section{Free Amino Acid Composition in the Honeydew of Asian Citrus Psyllid Nymphs}

The analysis of FAAs in the honeydew of ACP nymphs revealed that the contents of total amino acids in the honeydew secreted by the ACP nymphs from CLas-infected citrus plants were higher than that of ACP nymphs from CLas-free citrus plants, but the difference was not significant $(t=0.790, \mathrm{df}=2, P=0.512)$ (Figure 5A). In this study, we selected the same species of amino acids detected in the honeydew of CLas-positive and CLas-negative ACP populations for analysis and found that the CLas infection significantly affected the proportions of EAAs and NEAAs. Compared with the honeydew from ACP nymphs feeding on CLas-free citrus plants, the proportion of EAAs increased significantly $(t=61.518, \mathrm{df}=2, P<0.001)$, while the proportion of NEAAs decreased significantly in the honeydew of ACP nymphs feeding on CLas-infected citrus plants $(t=-6.654$, df $=2, P=0.022$ ) (Figure 5B). Results indicated that the efficiency of EAA absorption for ACP nymphs was increased, but the utilization was reduced when they fed on CLas-infected citrus plants. 


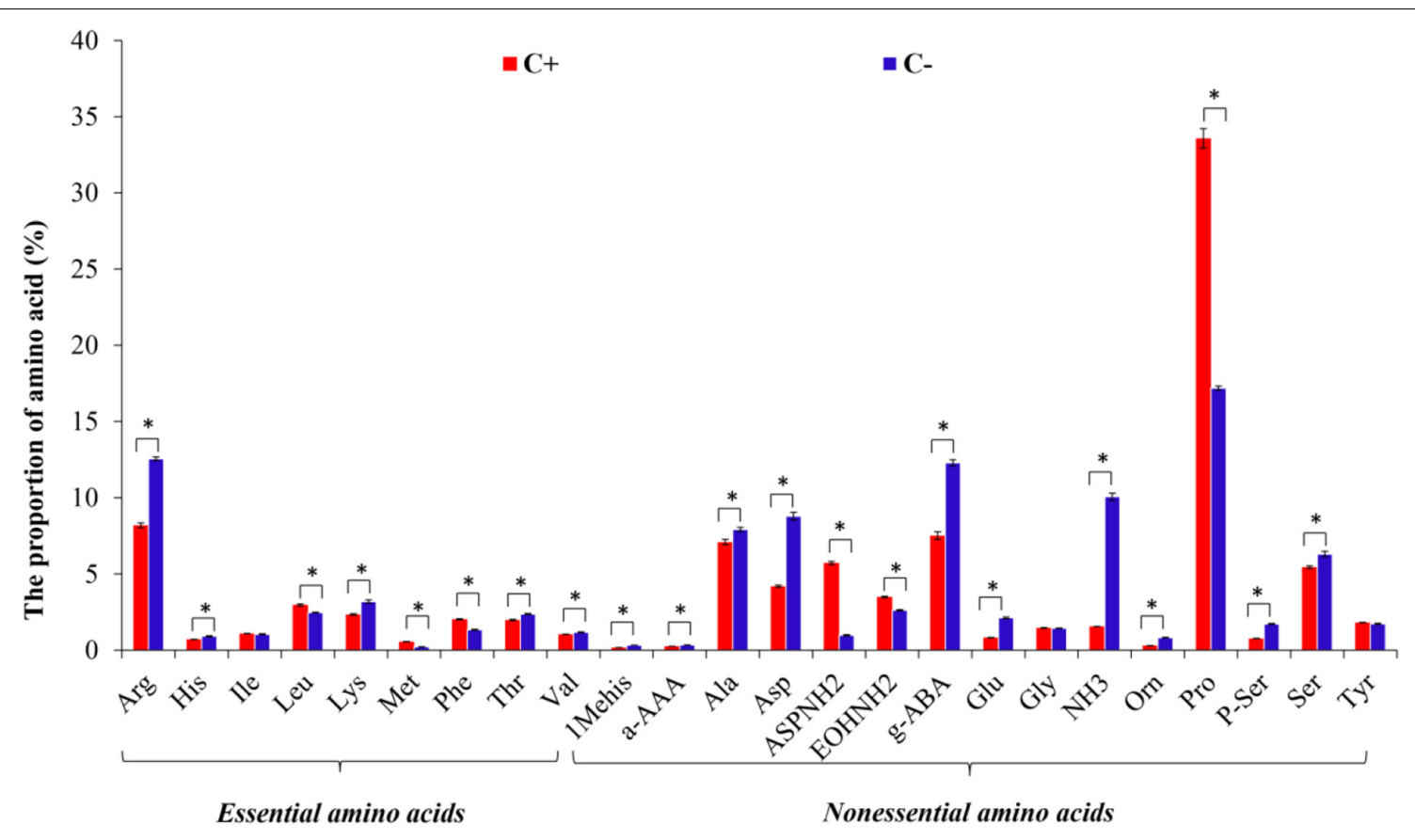

FIGURE 2 | The species of free amino acids (FAAs) in the phloem sap of citrus plants. "C+" and "C-" represent CLas-infected and CLas-free citrus plants, respectively. Values are represented as mean $\pm \mathrm{SE}$, and * over the bars means significant differences between groups at $P<0.05$.
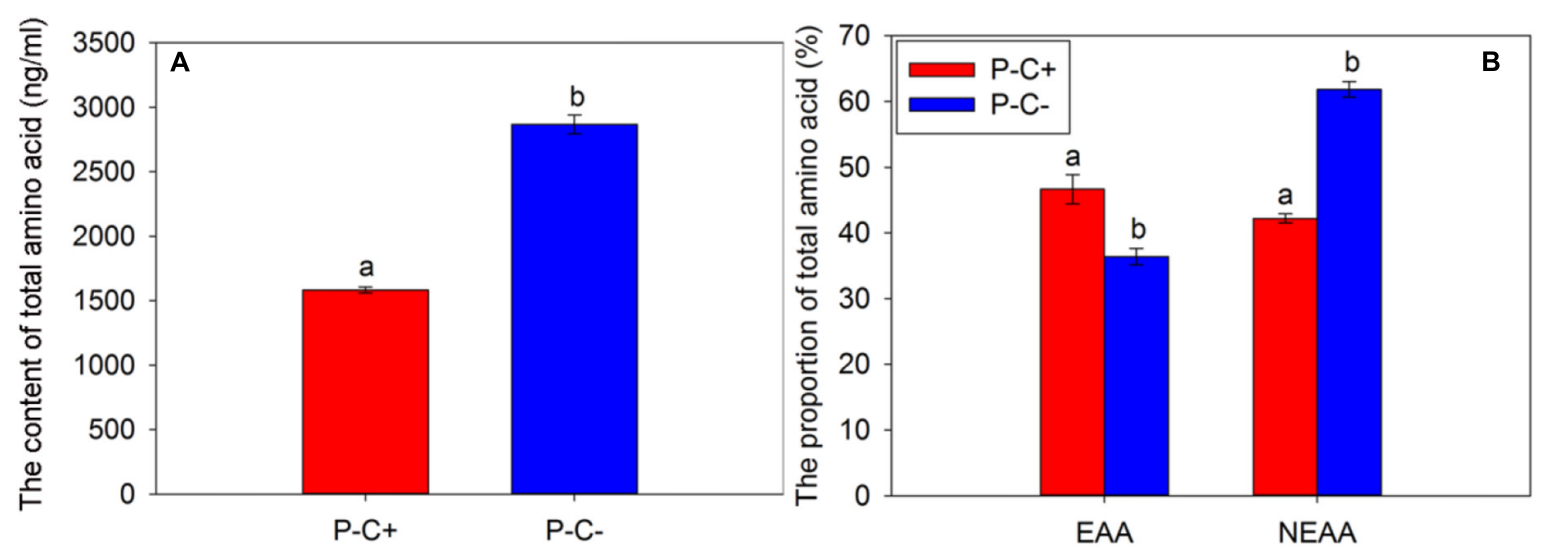

FIGURE 3 | The content of total amino acids (A) and the proportions of EAAs and NEAAs (B) in the Asian citrus psyllid (ACP) adults feeding on CLas-infected and CLas-free citrus plants. "P+" and "P-" represent CLas positive and negative psyllids, respectively, and "C+" and "C-" represent CLas-infected and CLas-free citrus pants, respectively. EAA, essential amino acids; NEAAs, non-essential amino acids. Values are represented as mean $\pm \mathrm{SE}$, and different letters over the bars mean significant differences between groups at $P<0.05$.

\section{Free Amino Acid Species in the Honeydew of Asian Citrus Psyllid Nymphs}

Similar to the FAAs in ACP nymphs, the same total of 25 species of FAAs was identified in the honeydew secreted by the ACP nymphs feeding on either CLas-free or CLas-infected citrus plants. The CLas infection significantly affected the EAAs proportion in ACP honeydew, mainly increasing the proportions of the EAAs such as His, Isoleucine (Ile), Leu, Lysine (Lys), Phenylalanine (Phe), Thr, Trp, and Valine (Val) while decreasing the proportions of NEAAs such as $\mathrm{ASPNH}_{2}$, Glutamic acid (Glu), and Pro in contrast to the proportions of other NEAAs, which were increased significantly $(P<0.05)$ (Figure 6).

\section{DISCUSSION}

Plants are frequently damaged by insect feeding and their associated vectored pathogens. These insect-vectored pathogens can alter numerous host plant factors, such as odors, visual, tactile, induced defense, sugars, FAA, and secondary metabolites 


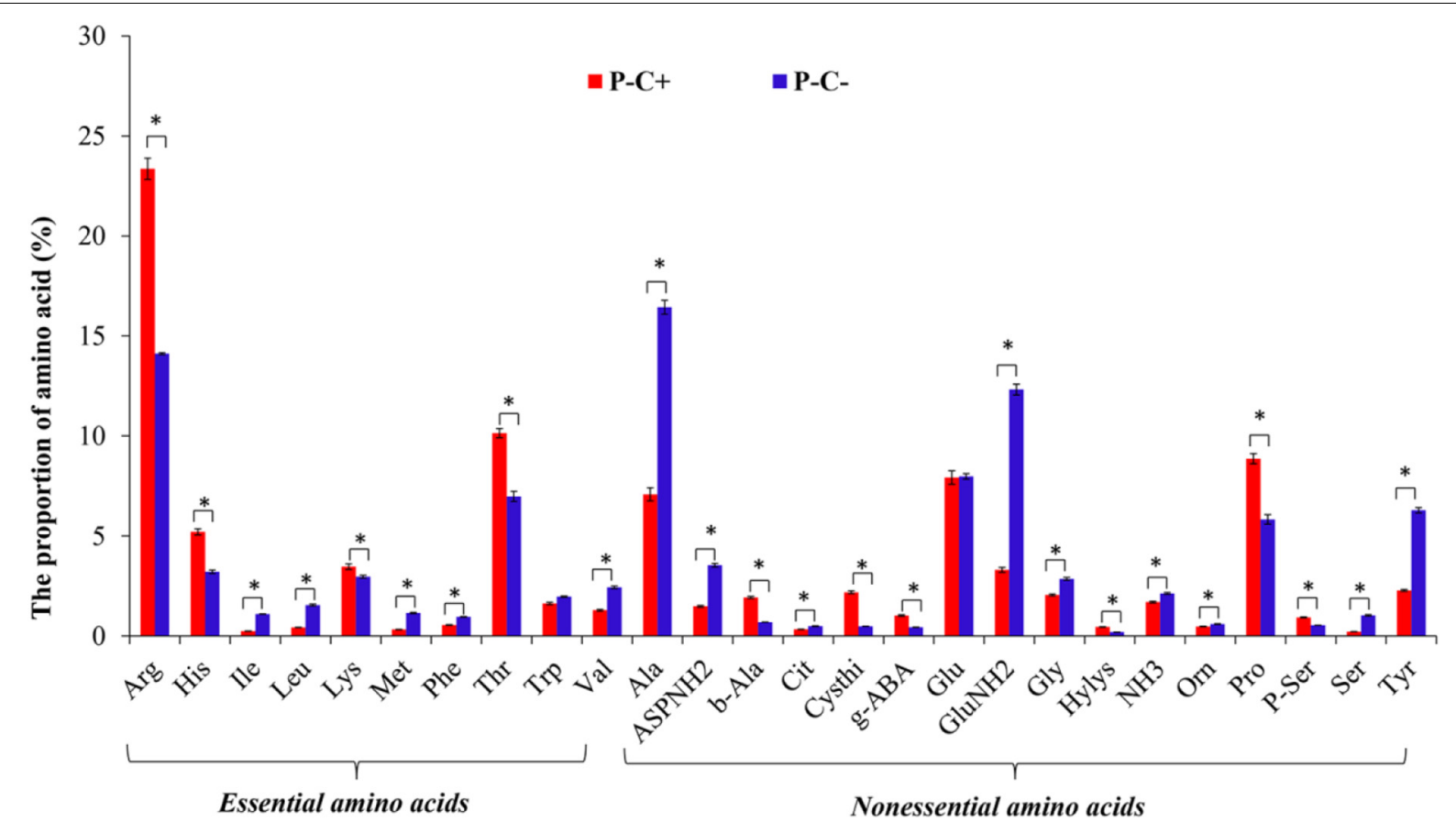

FIGURE 4 | The species of FAAs in the ACP adults feeding on CLas-infected and CLas-free citrus plants. "P+" and "P_" represent CLas positive and negative psyllids, respectively, and "C+" and "C-" represent CLas-infected and CLas-free citrus plants, respectively. Values are represented as mean \pm SE, and * over the bars means significant differences between groups at $P<0.05$.
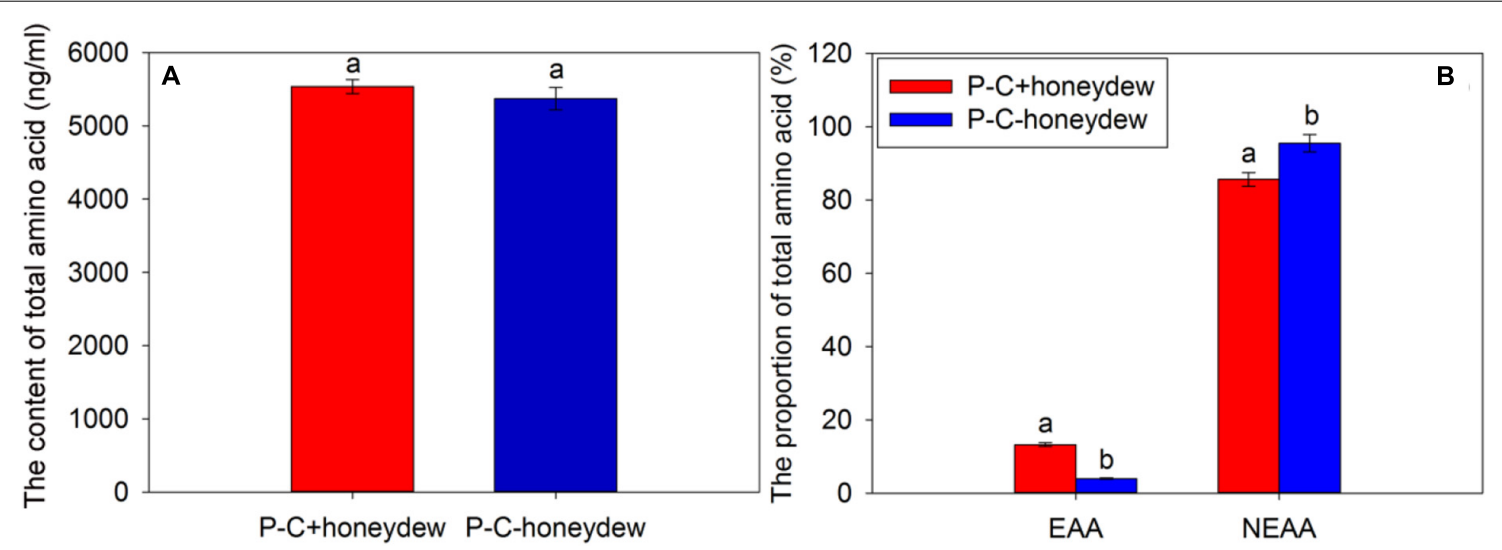

FIGURE $\mathbf{5}$ | The contents of total amino acids (A) and the proportions of EAAs and NEAAs (B) in honeydew excreted by ACP nymphs feeding on CLas-infected and CLas-free citrus plants. "P+" and "P-" represent CLas positive and negative psyllids, respectively, and "C+" and "C-" represent CLas-infected and CLas-free citrus plants, respectively. Values are represented as mean $\pm \mathrm{SE}$, and different letters over the bars mean significant differences between groups at $P<0.05$.

(Bosque-Pérez and Eigenbrode, 2011; Casteel et al., 2014; Mauck et al., 2014a,b). ACP is a hemipteran insect with a piercingsucking mouthpart that takes the phloem exudates of its host plant as its main food and nutrition source. In plant phloem exudates, the composition and content of amino acids are complex with FAAs being the main component (Sandström, 2000; Karley et al., 2002). In some early studies, a positive correlation was observed between the number of whitefly B. tabaci individuals and the amino acid content of their host plant (Crafts-Brandner, 2002). A subsequent study revealed that the concentrations of Ser, Ala, Pro, Phe, Glu, Asp, Arg, and Trp in the host plant played an important role in the survival rate of B. tabaci (Thompson, 2006). In our study, CLas infection significantly altered the content of total FAAs and the proportions of EAAs and NEAAs in the phloem sap of citrus plants, which is consistent with earlier studies on other interactions between plants and pathogens (Casteel et al., 2014; Su et al., 2015). In more detail, we found that CLas infection significantly reduced the FAA contents in the phloem of host plants, especially Ser, Ala, Glu, and Arg amino acids; these changes may affect 


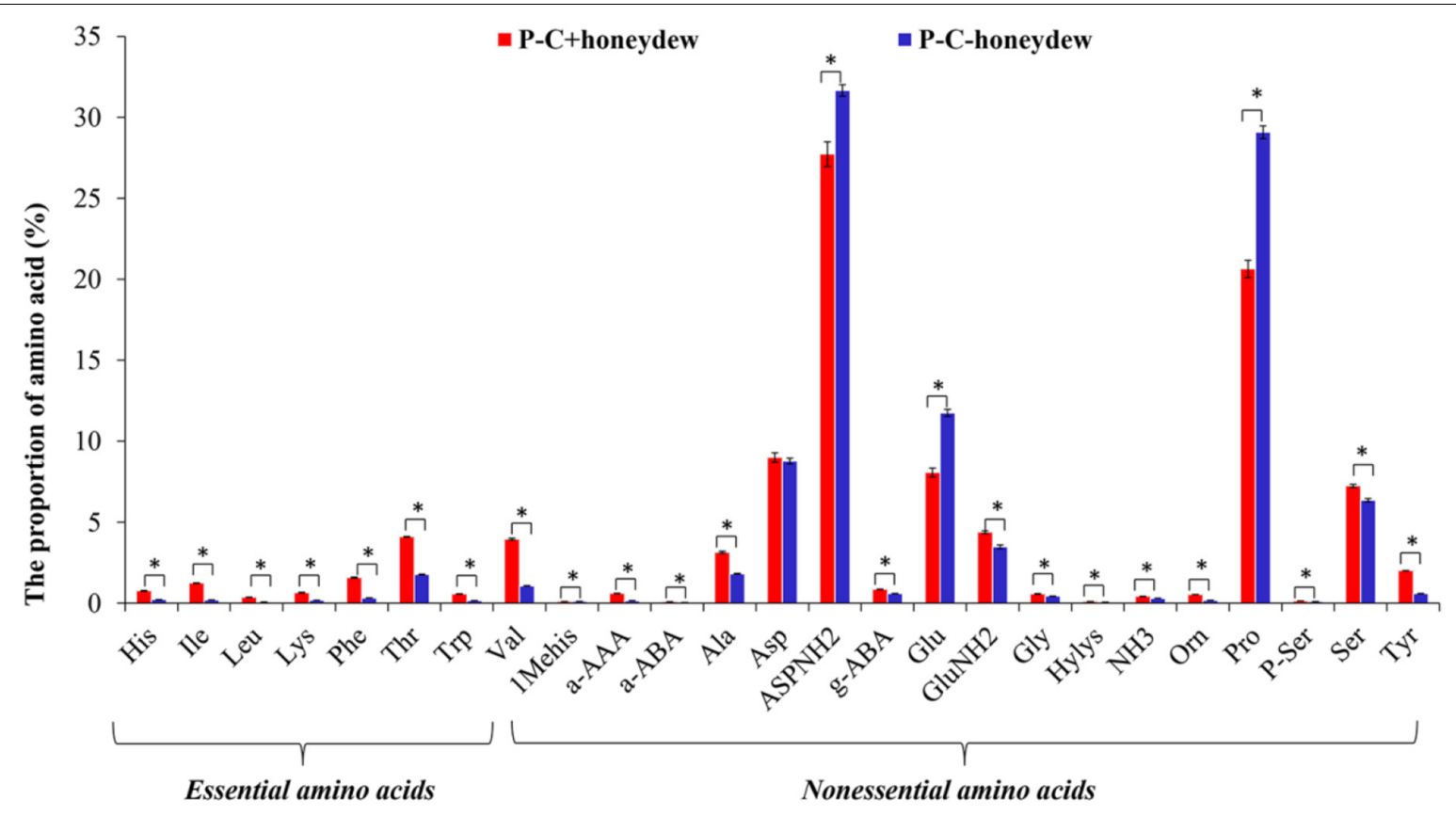

FIGURE 6 | The species of FAAs in honeydew excreted by ACP nymphs feeding on CLas-infected and CLas-free citrus plants. "P+" and "P-" represent CLas positive and negative psyllids, respectively, and " $\mathrm{C}+$ " and "C-" represent CLas-infected and CLas-free citrus plants, respectively. Values are represented as mean $\pm \mathrm{SE}$, and * over the bars means significant differences between groups at $P<0.05$.

the survival rate of ACP nymphs, supporting the previous findings of Ren et al. (2016).

In plant-pathogen-insect vector systems, the pathogen can directly or indirectly affect the insect behavior through the alteration of host plant physiology (Colvin et al., 2006; Stout et al., 2006; Belliure et al., 2010). Many insect herbivores prefer to select virus-infected plants because virus-infected plants often display better nutritional quality, more efficient absorption of nutrients, or repressed anti-herbivore defenses (Mauck et al., 2012; Wang, 2012; Ángeles-López et al., 2016). Our study further revealed that CLas infection increased the proportion of EAAs in ACP adults. It has been reported that the HLB pathogen lacks a complete aerobic respiratory pathway, so it needs an insect or plant host to provide it with EAAs and energy sources (Hijaz and Killiny, 2014). Further studies indicated that the HLB pathogen may manipulate the synthesis pathway of FAAs in ACP to supplement the intermediate products lacking in the tricarboxylic acid cycle (Killiny, 2018; Killiny et al., 2018). All these findings assist in the understanding of why the proportion of EAAs was higher than that of NEAAs in the ACP adults feeding on CLas-infected citrus plants.

It is well known that many amino acids, especially the EAAs obtained from diets, cannot be synthesized in insects but are necessary for their development and reproduction (Hansen and Moran, 2011; Boudko, 2012), for example, as phloem-feeders, aphids, and whiteflies absorb a diet that contains fairly high levels of FAAs (Buchanan et al., 2000). Stout et al. (2006) studied nutrition-related interactions between aphids and virus-infected plants where they found that the performance of aphids is often related to the nutritional quality of phloem sap. Rhopalosiphum padi and Sitobion avenae aphids showed both positive preference to and increased fecundities on cereal plants infected with Barley yellow dwarf virus compared to those on healthy cereals (Ferereset et al., 1989; Jiménez-Martínez et al., 2004a,b).

The development and reproduction of insects are not only correlated with the quality of diet but also related to their own utilization efficiency of nutrition (Montllor, 1989). The results of our study showed that CLas infection remarkably increased the mass proportions of Thr, His, Arg, and Pro in the ACP adults that fed on CLas-infected citrus plants, which indicated that the digestion and utilization efficiency of Pro by ACP adults was improved by CLas infection. Pro is the energy source of insects, and the oxidation of Pro through the Pro-Ala cycle has been found to be the major source of fuel for flight muscles in some insects (Crafts-Brandner, 2002). ACP adults from CLas-infected citrus have been found to be more active in migration between different citrus trees. The high efficiency of energy utilization of Pro may explain the energy source for frequent migration of ACP, and this stimulated dispersion and flight ability will facilitate the spread of CLas (Martini et al., 2015; Stelinski, 2019).

The content of the total amino acids was not significantly different between the honeydew secreted by the ACP nymphs feeding on CLas-infected and CLas-free citrus plants, but there were significant differences between the proportion and components of EAAs between the two types of honeydew. Taking this above finding, that is, CLas infection increased the proportion of EAAs in the honeydew of ACP nymphs, we proposed that the EAA utilization efficiency of those ACP nymphs feeding on CLas-infected citrus was much lower than those ACP nymphs feeding on CLas-free citrus plants. 
Mann et al. (2018) studied the effects of CLas infection on the development of midgut epithelial cells of $D$. citri. In this study, the midgut epithelial cells of nymphs exhibited a lower level of karyorrhexis in their early stage when they were infected with CLas. The midgut of an insect is the main site for the secretion of digestive enzymes, food digestion, and nutrient absorption. The efficiency decrease in the EAA absorption and utilization of the ACP nymphs feeding on CLas-infected citrus plants may be due to the fact that CLas infection could destroy the midgut epithelial cells of the ACP nymphs, so causing them to be unable to fully absorb and utilize nutrients. These unabsorbed nutrients are then excreted from the body in the form of honeydew. In our study, we found that CLas infection clearly reduced the proportion of Ser, Trp, and Ala in the honeydew of ACP nymphs. Thompson (2006) reported that the absorption of Ser, Ala, Glu, and Arg in host plants plays an important role in the survival rate of $B$. tabaci. Therefore, we speculated that the reason for the reduced survival rate of the nymphs may be related to Ser, Trp, and Ala absorption by the ACP nymphs not being fully utilized.

\section{CONCLUSION}

Our study has revealed that CLas infection alters the amino acid contents in the phloem sap of citrus plants, in ACP adults, and in the honeydew produced. CLas infection decreased the total amount of amino acids and the proportion of EAAs in host plants and also increased the proportion of EAAs in ACP but affected the utilization of EAAs by ACP. This information expands our knowledge concerning the nutritional requirements for plant phloem-limited pathogens and their insect vectors. CLas infection significantly affects the contents, proportions, and utilization efficiency of different amino acids in citrus plants and ACP nymphs, which together lead to a developmental pattern of ACP, which is more conducive to CLas transmission. The research results that were previously published by our laboratory in which ACP fed on citrus plants infected with HLB bacteria

\section{REFERENCES}

Ángeles-López, Y. I., Rivera-Bustamante, R. F., and Heil, M. (2016). Colonization by phloem-feeding herbivore overrides effects of plant virus on amino acid composition in phloem of chili plants. J. Chem. Ecol. 42, 85-988. doi: 10.1007/ s10886-016-0747-2

Belasque, J., Bassanezi, R. B., Yamamoto, P. T., Ayres, A. J., Tachibana, A., Violante, A. R. Jr., et al. (2010). Lesson from Huanglongbing management in São Paulo State, Brazil. J. Plant Pathol. 92, 285-302. doi: 10.4454/jpp.v92i2.171

Belliure, B., Janssen, A., Maris, P. C., Peters, D., and Sabelis, M. W. (2010). Herbivore arthropods benefit from vectoring plant viruses. Ecol. Lett. 8, 70-79. doi: 10.1111/j.1461-0248.2004.00699.x

Blua, M. J., Perring, T. M., and Madore, M. A. (1994). Plant virus-induced changes in aphid population development and temporal fluctuations in plant nutrients. J. Chem. Ecol. 20, 691-707. doi: 10.1007/BF02059607

Bosque-Pérez, N. A., and Eigenbrode, S. D. (2011). The influence of virus-induced changes in plants on aphid vectors: insights from luteovirus pathosystems. Virus Res. 159, 201-205. doi: 10.1016/j.virusres.2011.04.020

Boudko, D. Y. (2012). Molecular basis of essential amino acid transport from studies of insect nutrient amino acid transporters of the SLC6 family (NAT-SLC6). J. Insect Physiol. 58, 433-449. doi: 10.1016/j.jinsphys.2011.1 2.018 improved the development duration and fecundity of ACP, which supports the results of this study (Ren et al., 2016). The physiological mechanisms in relation to the importance of amino acids, nutritional requirements, and energy transformation are areas that still require further investigation.

\section{DATA AVAILABILITY STATEMENT}

The raw data supporting the conclusions of this article will be made available by the authors, without undue reservation.

\section{AUTHOR CONTRIBUTIONS}

L-HZ, S-LR, and B-LQ: conceived and designed the experiments. L-HZ, S-LR, Z-QS, P-PX, DO, and L-JW: performed the experiments. L-HZ, S-LR, and WS: analyzed the data. WS and B-LQ: contributed to reagents, materials, and analysis tools. L-HZ and B-LQ: wrote the manuscript. All authors contributed to the article and approved the submitted version.

\section{FUNDING}

This study was supported by the Laboratory of Lingnan Modern Agriculture Project (NT2021003), the NSFC-Guangdong Joint Research Fund (U1701231), the National High-Level Talent Special Support Plan (2020), the Science and Technology Program of Guangzhou, China (201807010019), and the Earmarked Fund for Guangdong Modern Agro-Industry Technology Research System (2021KJ108).

\section{ACKNOWLEDGMENTS}

We thank Andrew G. S. Cuthbertson (York, United Kingdom) for his critical comments on an earlier version of the manuscript.

Bové, J. M. (2006). Huanglongbing: a destructive, newly-emerging, century-old disease of citrus. J. Plant Pathol. 88, 7-37. doi: 10.4454/jpp.v88i1.828

Buchanan, B. B., Gruissem, W., and Jones, R. L. (eds) (2000). Biochemistry and Molecular Biology of Plants. Rockville, MD: American society of plant physiologist, 52-109.

Casteel, C. L., Yang, C., Nanduri, A. C., De Jong, H. N., Whitham, S. A., and Jander, G. (2014). The NIa-Pro protein of Turnip mosaic virus improves growth and reproduction of the aphid vector, Myzus persicae (green peach aphid). Pant. J. Cell Mol. Biol. 77, 653-663. doi: 10.1111/tpj.12417

Colvin, J., Omongo, C. A., Govindappa, M. R., Stevenson, P. C., and Muniyappa, V. (2006). Host-plant viral infection effects on arthropod-vector population growth, development and behaviour: management and epidemiological implications. Adv. Virus Res. 67, 419-452. doi: 10.1016/S0065-3527(06)67011-5

Coy, M. R., Hoffmann, M., Gibbard, H. N. K., Kuhns, E. H., Pelz-Stelinski, K. S. A., and Stelinski, L. L. (2014). Nested-quantitative PCR approach with improved sensitivity for the detection of low titer levels of Candidatus Liberibacter asiaticus in the Asian citrus psyllid, Diaphorina citri Kuwayama. J. Microbiol. Meth. 102, 15-22. doi: 10.1016/j.mimet.2014.04.007

Crafts-Brandner, S. J. (2002). Plant nitrogen status rapidly alters amino acid metabolism and excretion in Bemisia tabaci. Insect Physiol. 48, 33-41. doi: 10.1016/S0022-1910(01)00140-8

Ding, J.-H. (1990). Utilization of free amino acids by brown plant hopper. Chin. Bull. Entomol. 65-67. 
Ferereset, A., Lister, R. M., Araya, J. E., and Foster, J. E. (1989). Development and reproduction of the English grain aphid (Homoptera: Aphididae) on wheat cultivars infected with barley yellow dwarf virus. Environ. Entomol. 18, 388-393. doi: 10.1093/ee/18.3.388

Garnier, M., Bové, J., Cronje, C., Sanders, G., Korsten, L., and Le Roux, H. (2000). "Presence of "Candidatus Liberibacter africanus" in the Western Cape province of South Africa," in Proceedings of the 14th Conference of International Organization of Citrus Virologists, Riverside, CA, 369-372. Retrieved from https: //escholarship.org/uc/item/2sz5q3w7.

Guo, L., Su, Q., Yin, J., Yang, Z., Xie, W., Wang, S. L., et al. (2019). Amino acid utilization may explain why Bemisia tabaci $\mathrm{Q}$ and B differ in their performance on plants infected by the Tomato yellow leaf curl virus. Front. Physiol. 10:489. doi: 10.3389/fphys.2019.00489

Hansen, A., and Moran, N. A. (2011). Aphid genome expression reveals hostsymbiont cooperation in the production of amino acids. Proc. Natl. Acad. Sci. U. S. A. 108, 49-54. doi: 10.1073/pnas. 1013465108

Hijaz, F., and Killiny, N. (2014). Collection and chemical composition of phloem sap from Citrus sinensis L. Osbeck (Sweet Orange). PLoS One 9:e101830. doi: 10.1371/journal.pone.0101830

Holopainen, J., Kainulainen, P., and Oksanen, J. (1997). Growth and reproduction of aphids and levels of free amino acids in Scots pine and Norway spruce in an open-air fumigation with ozone. Global Change Biol. 3, 139-147. doi: 10.1046/j.1365-2486.1997.00067.x

Jagoueix, S., Bove, J. M., and Garnier, M. (1994). The phloem limited bacterium of greening disease of citrus is a member of the a subdivision of the Proteobacteria. Int. J. Syst. Bacteriol. 44, 379-386. doi: 10.1099/00207713-44-3-379

Jiménez-Martínez, E. S., Bosque-Pérez, N. A., Berger, P. H., Zemetra, R. S., Ding, H., and Eigen-brode, S. D. (2004a). Volatile cues influence the response of Rhopalosiphum padi (Homoptera: Aphididae) to barley yellow dwarf virusinfected transgenic and un-transformed wheat. Environ. Entomol. 33, 12071216. doi: 10.1603/0046-225X-33.5.1207

Jiménez-Martínez, E. S., Bosque-Pérez, N. A., Berger, P. H., and Zemetra, R. S. (2004b). Life history of the bird cherry-oat aphid, Rhopalosiphum padi (Homoptera: Aphididae), on transgenic and untransformed wheat challenged with barley yellow dwarf virus. J. Econ. Entomol. 97, 203-212. doi: 10.1603/ 0022-0493-97.2.203

Jiu, M., Zhou, X. P., Tong, L., Xu, J., Yang, X., Wan, F. H., et al. (2007). Vectorvirus mutualism accelerates population increase of an invasive whitefly. PLoS One 2:e182. doi: 10.1371/journal.pone.0000182

Karley, A. J., Douglas, A. E., and Parker, W. E. (2002). Amino acid composition and nutritional quality of potato leaf phloem sap for aphids. J. Exp. Biol. 205, 3009-3018. doi: 10.1242/jeb.205.19.3009

Killiny, N. (2018). Generous hosts: why the larvae of greater wax moth, Galleria mellonella is a perfect infectious host model? Virulence 9, 860-865. doi: 10. 1080/21505594.2018.1454172

Killiny, N., Nehela, Y., Hijaz, F., and Vincent, C. I. (2018). A plant pathogenic bacterium exploits the tricarboxylic acid cycle metabolic pathway of its insect vector. Virulence 9, 99-109. doi: 10.1080/21505594.2017.1339008

Mann, M., Fattah-Hosseini, S., Ammar, E. D., Stange, R., Warrick, E., Sturgeon, K., et al. (2018). Diaphorina citri nymphs are resistant to morphological changes Induced by "Candidatus Liberibacter asiaticus" in midgut epithelial cells. Infect. Immun. 86, e889-e881. doi: 10.1128/iai.00889-17

Martini, X., Hoffmann, M., Coy, M. R., Stelinski, L. L., and Pelz-Stelinski, K. S. (2015). Infection of an insect vector with a bacterial plant pathogen increases its propensity for dispersal. PLoS One 10:e0129373. doi: 10.1371/journal.pone. 0129373

Mauck, K., Bosque-Pérez, N. A., Eigenbrode, S. D., Moraes, C. M. D., and Mescher, M. C. (2012). Transmission mechanisms shape pathogen effects on host-vector interactions: evidence from plant viruses. Funct. Ecol. 26, 1162-1175. doi: $10.2307 / 23326861$

Mauck, K. E., De Morases, C. M., and Mescher, M. C. (2014a). Biochemical and physiological mechanisms underlying effects of Cucumber mosaic virus on host-plant traits that mediate transmission by aphid vectors. Plant Cell Environ. 37, 1427-1439. doi: 10.1111/pce.12249

Mauck, K. E., De Moraes, C. M., and Mescher, M. C. (2014b). Evidence of local adaptation in plant virus effects on host-vector interactions. Integr. Comp. Biol. 54, 193-209. doi: 10.1093/icb/icu012

Montllor, C. B. (1989). "The influence of plant chemistry on aphid feeding behavior”, in Insect Plant Interactions, Vol. 3, ed. E. Bernays (Boca Raton, FL: CRC Press), 125-173. doi: 10.1201/9780203711699
Onagbola, E. O., Rouseff, R. L., Smoot, J. M., and Stelinski, L. L. (2011). Guava leaf volatiles and dimethyl disulphide inhibit response of Diaphorina citri Kuwayama to host plant volatiles. J. Appl. Entomol. 135, 404-414. doi: 10.1111/ j.1439-0418.2010.01565.x

Pan, H., Su, Q., Jiao, X., Zhou, L., Liu, B., Xie, W., et al. (2013). Relative amount of symbionts in Bemisia tabaci (Gennadius) Q changes with host plant and establishing the method of analyzing free amino acid in B. tabaci. Comm. Integr. Biol. 6:e23397. doi: 10.4161/cib.23397

Ren, S. L., Li, Y. H., Zhou, Y. T., Xu, W. M., and Qiu, B. L. (2016). Effects of Candidatus Liberibacter asiaticus on the fitness of the vector Diaphorina citri. J. Appl. Microbiol. 121, 1718-1726. doi: 10.1111/jam.13302

Roistacher, C. N. (1996). "The economics of living with citrus diseases: huanglongbing (greening) in Thailand," in Proceedings of $13^{\text {th }}$ Conference of International Organization of Citrus Virologists, Riverside CA, 279-285. Retrieved from https://escholarship.org/uc/item/3kg8m6jr.

Sandström, J. (2000). Nutritional quality of phloem sap in relation to host plantalternation in the bird cherry-oat aphid. Chemoecology 10, 17-24. doi: 10.1007/ s000490050003

Stelinski, L. L. (2019). Ecological aspects of the vector-borne acterial disease, Citrus greening (Huanglongbing): dispersal and host use by Asian Citrus Psyllid, Diaphorina Citri Kuwayama. Insects 10:208. doi: 10.3390/insects10070208

Stout, M. J., Thaler, J. S., and Thomma, B. P. H. J. (2006). Plant-mediated interactions between pathogenic microorganisms and herbivorous arthropods. Annu. Rev. Entomol. 51, 663-689. doi: 10.1146/annurev.ento.51.110104.151117

Su, Q., Preisser, E. L., Zhou, X. M., Xie, W., Liu, B. M., Wang, S. L., et al. (2015). Manipulation of host quality and defense by a plant virus improves performance of whitefly vectors. J. Econ. Entomol. 108, 11-19. doi: 10.1093/jee/tou012

Texeira, D. C., Ayres, J., Kitajima, E. W., Danet, L., Jagoueixeveillard, S., Saillard, C., et al. (2005). First report of a huanglongbing-like disease of citrus in sao paulo state, brazil and association of a new liberibacter species, "Candidatus Liberibacter americanus", with the disease. Plant Dis. 6, 89-107. doi: 10.1094/ PD-89-0107A

Thompson, W. M. O. (2006). Influence of amino acids on cassava biotype Bemisia tabaci (Gennadius) (Homoptera: Aleyrodidae) when feeding on an artificial system. J. Entomol. 3, 198-203. doi: 10.3923/je.2006.198.203

Tsai, J., Chen, Z., Shen, C., and Jin, K. (1988). "Mycoplasmas and fastidious vascular prokaryotes associated with tree diseases in China," in Tree Mycoplasmas and Mycoplasma Diseases, ed. C. Hiruki (Edmonton, AB: University Alberta Press), 69-97.

Walter, A. J., and Difonzo, C. D. (2014). Soil potassium deficiency affects soybean phloem nitrogen and soybean aphid populations. Environ. Entomol. 36, 26-33. doi: 10.1603/0046-225X(2007)36[26:SPDASP]2.0.CO;2

Wang, J. (2012). The Nutrition-Related Mechanisms Underlying TYLCCNV-MEAM 1 Whitefly-Tobacco Plant Interactions. Ph.D. thesis. Hangzhou, Zhejiang University. (in Chinese).

Wang, Y., Zhou, L., Yu, X., Stover, E., Luo, F., and Duan, Y. (2016). Transcriptome profiling of Huanglongbing (HLB) tolerant and susceptible citrus plants reveals the role of basal resistance in HLB tolerance. Front Plant Sci. 7:933. doi: 10.3389/fpls.2016.00933

Zhao, X. P., Liu, K. W., Yang, S. Z., Yu, D., and Ou, Z. (2001). Studies on free amino acids of Ericerus pela and its host plant. Entomol. Know. 6, $456-459$.

Conflict of Interest: The authors declare that the research was conducted in the absence of any commercial or financial relationships that could be construed as a potential conflict of interest.

Publisher's Note: All claims expressed in this article are solely those of the authors and do not necessarily represent those of their affiliated organizations, or those of the publisher, the editors and the reviewers. Any product that may be evaluated in this article, or claim that may be made by its manufacturer, is not guaranteed or endorsed by the publisher.

Copyright () 2021 Zhang, Ren, Su, Xu, Ou, Wang, Sang and Qiu. This is an openaccess article distributed under the terms of the Creative Commons Attribution License (CC BY). The use, distribution or reproduction in other forums is permitted, provided the original author(s) and the copyright owner(s) are credited and that the original publication in this journal is cited, in accordance with accepted academic practice. No use, distribution or reproduction is permitted which does not comply with these terms. 